\title{
Discrete-Time Financial Planning Models Under Loss-Averse Preferences
}

\author{
Arjen Siegmann \\ Department of Finance, Vrije Universiteit Amsterdam, De Boelelaan 1105, NL, Amsterdam, The Netherlands, and \\ Research Department, Netherlands Central Bank (DNB), asiegmann@feweb.vu.nl \\ André Lucas \\ Department of Finance, Vrije Universiteit Amsterdam, De Boelelaan 1105, NL, Amsterdam, The Netherlands, and \\ Tinbergen Institute, Amsterdam, The Netherlands, alucas@feweb.vu.nl
}

\begin{abstract}
We consider a dynamic asset allocation problem formulated as a mean-shortfall model in discrete time. A characterization of the solution is derived analytically under general distributional assumptions for serially independent risky returns. The solution displays risk taking under shortfall, as well as a specific form of time diversification. Also, for a representative stock-return distribution, risk taking increases monotonically with the number of decision moments given a fixed horizon. This is related to the well-known casino effect arising in a downside-risk and expected return framework. As a robustness check, we provide results for a modified objective with a quadratic penalty on shortfall. An analytical solution for a single-stage setup is derived, and numerical results for the two-period model and time diversification are provided.
\end{abstract}

Subject classifications: multistage stochastic programming; downside risk; asset/liability management; time diversification.

Area of review: Financial Engineering.

History: Received November 2002; revision received February 2004; accepted April 2004.

\section{Introduction}

Despite extensive research in the area of stochastic programming, analytic solutions to multistage stochastic programs are rare. The main contribution of this paper is twofold. First, we analytically characterize the solution to a basic multistage financial planning model and study its robustness. Our setup shares the main characteristics with more elaborate applied models that are widely used in the financial industry. As such, our second contribution is to highlight some of the key mechanisms and potential pitfalls of practical financial planning models with downside-risk measures.

Over the past decade, we have witnessed a growing literature on financial planning models. Such models can assist financial institutions like pension funds, insurance companies, and banks in their asset/liability management (ALM), as illustrated in the book by Ziemba and Mulvey (eds.) (1998). The key component in these models concerns the trade-off between risk and return. It is therefore of paramount importance which risk measure is put into the model. Traditionally, the variance or standard deviation has been the prominent measure of risk, with the main (and perhaps only) advantage being its computational simplicity. As argued by Sortino and Van der Meer (1991), however, the variance is an inadequate measure of risk in many practical circumstances. The main criticism to the use of the variance is its symmetric nature, whereas risk is typically perceived as an asymmetric phenomenon.
Asymmetric or downside-risk measures are generally more difficult to work with, both analytically and computationally. Given the current state of computer technology, however, their use in both theoretical and empirical financial planning models has increased rapidly.

Downside-risk measures are currently used extensively in the area of ALM. Recent research typically incorporates these risk measures in a multistage stochastic programming (MSP) approach, as in Consigli and Dempster (1998), Mulvey and Thorlacius (1998), Cariño et al. (1994), Boender (1997), and Dert (1998). The main advantage of the MSP approach relative to the more traditional static meanvariance oriented approach is that the explicit dynamic nature of financial decisions can better be taken into account. For example, a decision now may be followed by recourse actions in the future. Moreover, different preferences and (dynamic) constraints can be modeled directly. As a result, the MSP approach generally produces significant improvements over static mean-variance-based decisions. These improvements can be exploited when the MSP model is implemented in practice, as in Cariño et al. (1994).

The interaction between the formulation of an MSP model and the resulting decisions is generally badly understood. In this paper, we solve a basic model analytically and present the solution as decision rules in feedback form. These rules have a nice economic interpretation. Parametric decision rules have also been derived for models formulated in continuous time as in Merton (1969) and Sethi (1998) for 
the consumption/investment problem and Ingersoll (1987) for the general portfolio optimization problem. See also Basak and Shapiro (2001), who optimize expected utility of terminal wealth under a constraint on downside risk. By contrast, our model is set in discrete time, for which few analytic results are available.

The solution to our model is found to be a set of V-shaped rules: risk taking increases for both increasingly high and increasingly low wealth levels. The exact shape depends on the return distributions and the time to the horizon. Moreover, assuming independently distributed returns across time, we find increasing risk-taking behavior when the distance to the horizon increases. The increased risk taking for low wealth levels may appear undesirable from a normative or practitioner point of view. We show, however, that this phenomenon is surprisingly robust over variations in the specification. As a side result of our paper, these findings lead to some fundamental questions regarding the typical use of loss-averse preferences in financial optimization problems.

The remainder of this paper is organized as follows. In $\S 2$, the multistage model is introduced. Section 3 gives the solution to the multistage model and the corresponding optimal decision rules. It also gives a number of interesting consequences that follow from the solution. Section 4 gives some generalizations of the model using an alternative objective function. Concluding remarks are given in $\S 5$. The appendix contains the proofs.

\section{A Downside-Risk Model}

In formulating a simple downside-risk model, we draw on a number of existing articles that formulate variants of downside-risk models. For ease of exposition, we first give the formulation of our basic ALM model and then explain how it is representative of existing empirical models.

Let $W_{t}$ denote wealth at time $t=0, \ldots, T$. At each time $t$ there are two investment opportunities: a risk-free asset with certain return $r_{f}$ in each period, and a risky asset with uncertain return $u_{t}$ over period $t$. Restricting the investment categories to only two assets may appear restrictive at first. However, as shown by Merton (1990) for example, if two-fund separation holds in an economy, all efficient asset allocations are completely covered by investments in the risk-free asset and the market portfolio only. In this case, our $u_{t}$ would represent the return on the market portfolio, i.e., a portfolio that holds all available securities in proportion to their market values. See Cass and Stiglitz (1970) for a general discussion of separation theorems. An important feature of the model is that the exact distribution of $u_{t}$ can be left unspecified.

With the two investment categories given, wealth evolves as

$W_{t+1}=W_{t} \cdot r_{f}+X_{t} \cdot\left(u_{t+1}-r_{f}\right), \quad t=0, \ldots, T-1$,

where $X_{t}$ is the amount invested in the risky asset at the beginning of period $t$. The returns $u_{1}, \ldots, u_{T}$ are independent, though not necessarily identically distributed with absolute continuous distribution functions $G_{t+1}(\cdot)$ on $[0, \infty)$. We assume that $\mathbb{E}_{t}\left[u_{t+1}\right]>r_{f}$, with $\mathbb{E}_{t}(\cdot)=$ $\int \cdot d G_{t+1}$, i.e., the expected return on the risky asset always exceeds the risk-free return.

Incorporating a downside-risk measure relative to a benchmark level of final wealth $W_{T}^{B}$, the objective function is given by

$\max _{X_{0}, \ldots, X_{T-1}} \mathbb{E}_{0}\left[W_{T}\right]-\lambda \cdot \mathbb{E}_{0}\left[\left(W_{T}^{B}-W_{T}\right)^{+}\right]$,

with $\lambda>0$ the risk-aversion parameter, and $(y)^{+}$denoting the maximum of 0 and $y$. In words, the objective (2) aims to maximize expected final wealth with a penalty of $\lambda$ for each dollar shortfall below a reference wealth level $W_{T}^{B}$ at time $T$.

Model (2) resembles the formulation of the stochastic programming example for a financial planning model in Birge and Louveaux (1997). There the setting is given by parents who wish to provide for a child's college education $T$ years from now. $W^{B}$ is the tuition goal and $\lambda$ the (total) cost of borrowing if the goal is not met. The example is used to show how stochastic programming can be used to numerically solve the problem. This paper shows that an analytic solution can be obtained.

Our utility function with $W^{B} \equiv W_{0}$ is discussed in Sharpe (1998) to show that the then-used Morningstar rating for mutual fund performance evaluation could lead to extreme investment strategies. This also follows directly from our subsequent analysis. However, Sharpe's critique only holds for $W_{0} \equiv W^{B}$, which in our setting can be considered a special case. Also, our robustness analysis in $\$ 4$ shows that for a quadratic penalty, this behavior disappears.

Bertsimas et al. (2004) analyze the properties and use of expected shortfall as a risk measure, showing how the mean-shortfall approach as in (2) can have advantages over mean variance. Many studies consider the numerical properties of problems with a comparable objective. Hiller and Eckstein (1993) use the same objective function as (2) in a stochastic dedication model for fixed-income portfolios. (2) also resembles the objective function of Cariño et al. (1994), albeit that they use a piecewise linear penalty function in terms of the expected loss, where we have a linear one. In addition, they argue that in the context of banks and insurance companies, risk measures as in (2) can easily be justified, as these companies are faced with specific costs if reserves fall below critical threshold levels. The risk measure in (2) in terms of returns is used by Rockafellar and Uryasev (2000) in a static stochastic portfolio optimization. Basak and Shapiro (2001) also use expected shortfall as the risk measure in a constraint for a continuous-time dynamic portfolio optimization model.

Almost exactly the same objective is often used in practical ALM studies for pension funds, and fixed income investment studies for corporate treasuries. ALM is foremost a practical science. Cariño et al. (1994) describe an implemented ALM model where downside risk is incorporated 
as a stepped penalty function accommodating for the specific thresholds at which risks become effective. Boender (1997) describes the details of an ALM model that is used empirically for advising defined benefit pension funds. His objective function contains a quadratic penalty on shortfall. Both as a robustness check and because quadratic penalty functions are also often used in practice, we also study how our results for (2) generalize to a situation with quadratic shortfall in $\$ 4$.

An essential feature of the objective in (2) is the need to specify the reference point $W^{B}$. It has the advantage of making risk explicit and for several institutional settings there is a natural candidate. In the case of defined benefit pension funds, $W_{t}$ represents the pension fund's asset level and $W^{B}$ the present value of liabilities.

A number of potential drawbacks of the mean-shortfall model in (2) can be seen already. First, as the objective is piecewise linear, it gives rise to locally risk-neutral behavior. In a complete markets setting, this may give rise to unbounded solutions; see Dert and Oldenkamp (2000) and $\S 3.5$. This is generally undesirable from a normative point of view and empirical models usually incorporate several types of institutional constraints to prevent this effect, e.g., limited investment in specific assets or a nonnegativity constraint on wealth. The incorporation of such constraints, however, only obscures the main drivers and possible pitfalls of financial planning models based on expected return and downside risk. Therefore, it is important to stick to our simple objective function to highlight the model's basic mechanisms. Local risk neutrality may be removed by imposing convex penalties on shortfall as in $\$ 4$ and concave utilities to gains. In a discrete-time framework with incomplete markets, however, this quickly becomes intractable.

A second property of (2) is that situations of shortfall are not explicitly excluded. Again, this may appear undesirable from a normative perspective. On closer inspection, however, the current setup allows for some interesting new insights. First, it allows us to explicate the incentives for risk taking provided by mean-downside-risk models in situations of initial shortfall. This is not always possible if an explicit constraint on shortfall is included. In that case, a feasible solution may often not exist. Second, the situation of an initial negative surplus, though possibly normatively undesirable, is empirically relevant. Even if explicit probabilistic constraints on shortfall are imposed at a certain stage of the planning process, a situation of shortfall may easily arise in subsequent stages. This is due to the fact that shortfall generally has a strictly positive, though possibly small, probability at the optimum. The bear stock markets over the early 2000s have shown that shortfall may indeed (nearly) arise at many defined benefit pension funds and insurance companies.

\section{Results}

Using the special structure of the model, we obtain the complete solution to (2) up to a set of $2 * T$ parameters that depend directly on the model parameters and the probability distributions of the risky returns $u_{t+1}$. We present the solution and subsequently discuss its financial implications for (i) the role and interpretation of $\lambda$, (ii) risk taking in a shortfall position, and (iii) a result on time diversification that fits in a behavioral finance perspective.

\subsection{Solution to the Multistage Model}

Define $W_{t}^{B}=W_{T}^{B} / r_{f}^{T-t}$ as the risk-free discounted value of benchmark wealth at time $t$, and $S_{t}=W_{t}-W_{t}^{B}$ as the surplus. The next theorem characterizes the solution to (2).

THEOREM 3.1. If there is a bounded solution to the optimization problem in (2) and (1), then it is given by

$X_{t}^{*}=\frac{r_{f}}{r_{f}-\bar{u}_{t+1}^{*}} \cdot S_{t}, \quad t=0, \ldots, T-1$,

where $\bar{u}_{t+1}^{*}$ is smaller than $r_{f}$ for a positive surplus and larger than $r_{f}$ for a negative surplus, so that $X_{t}^{*} \geqslant 0$. Specifically, $\bar{u}_{t+1}^{*}$ is one out of two possible $\bar{u}_{t}$ s that solve

$\mathbb{E}_{t}\left[u_{t+1}-r_{f}\right]=\lambda_{t} \cdot \mathbb{E}_{t}\left[\left(r_{f}-u_{t+1}\right) \cdot I_{\left\{u_{t+1} \leqslant \bar{u}\right\}}\right]$.

The two solutions to (4) are labeled $\bar{u}_{t+1}^{+}<r_{f}$ and $\bar{u}_{t+1}^{-}>r_{f}$. Further, $\lambda_{t}$ is defined by

$\lambda_{t}=\frac{\lambda \cdot\left(p_{t+1}^{-}-p_{t+1}^{+}\right)}{1+\lambda \cdot p_{t+1}^{+}}$,

where for $k=t+1, \ldots, T-1, p_{k}^{+}$and $p_{k}^{-}$are defined recursively by

$p_{k}^{-}=p_{k+1}^{-} \cdot G_{k+1}\left(\bar{u}_{k+1}^{-}\right)+p_{k+1}^{+}\left(1-G_{k+1}\left(\bar{u}_{k+1}^{-}\right)\right)$,

$p_{k}^{+}=p_{k+1}^{-} \cdot G_{k+1}\left(\bar{u}_{k+1}^{+}\right)+p_{k+1}^{+}\left(1-G_{k+1}\left(\bar{u}_{k+1}^{+}\right)\right)$,

and $p_{T}^{-} \equiv 1, p_{T}^{+} \equiv 0$.

Proof. See the appendix.

Equation (3) shows that $X_{t}^{*}$, the optimal amount invested in the risky asset, is a piecewise linear function of the surplus $S_{t}$. There is one "kink" at zero surplus. The slope of the decision rule is only affected by the sign of the surplus, which determines $\bar{u}_{t+1}^{*}$ through Equation (4). Note that the right-hand side of (4) is unimodal in $\bar{u}$. The maximum is reached at $\bar{u}=r_{f}$. If

$$
\begin{aligned}
\lambda_{t} \cdot \mathbb{E}_{t}\left[\left(r_{f}-u_{t+1}\right) \cdot I_{\left\{u_{t+1} \leqslant r_{f}\right\}}\right]>\mathbb{E}_{t}\left[u_{t+1}-r_{f}\right], & \\
& t=0, \ldots, T-1,
\end{aligned}
$$

then (4) has two distinct solutions: one smaller and one larger than $r_{f}$. Consequently, (8) is a sufficient condition for having a bounded solution. Effectively, it puts a lower bound on the risk-aversion parameter $\lambda$. The economic interpretation of the lower bound is that if $\lambda$ is too small, the marginal expected loss of an extra unit invested in the risky asset is always smaller than the risk premium $\mu-r_{f}$. 
Figure 1. Solving the first-order condition.

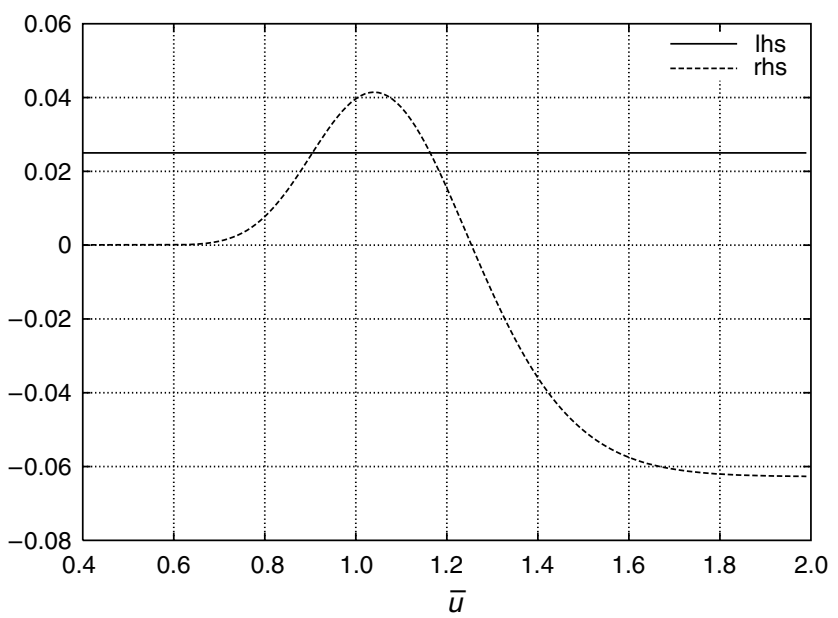

Notes. The solid line "lhs" represents $\mathbb{E}\left[u_{t+1}-r_{f}\right] / \lambda_{t}$, the left-hand side of (4) scaled by $\lambda_{t}$. The dashed curve "rhs" represents $\mathbb{E}_{t}\left[\left(r_{f}-u_{t+1}\right)\right.$. $\left.I_{\left\{u_{t+1} \leqslant \bar{u}\right\}}\right]$, the right-hand side of (4), also scaled by $\lambda_{t}$. The points of intersection are the $\bar{u}$ s that solve the first-order condition. The riskfree rate $r_{f}$ is $1.04, \lambda_{t}=2.4$, and the uncertain return $u$ is distributed log-normal $(0.085,0.16)$, representing a typical stock return with a mean return of $10 \%$ and standard deviation of $17 \%$.

Hence, without the lower bound investment in the risky asset can be infinite, which we want to exclude. Note that without a specification for $G(\cdot)$, an expression of condition (8) in primitives is not possible.

Under (8), solving Equation (4) can be visualized as in Figure 1. It boils down to evaluating the conditional first moments of $G_{t+1}(\cdot)$. This can be done very efficiently. If (8) is not met, either $\bar{u}_{t+1}^{*}=r_{f}$ or (4) has no solution, corresponding to the horizontal solid line in Figure 1 just touching or lying completely above the dashed curve. In both cases, the optimal decision $X_{t}^{*}$ in (3) is unbounded.

From the definition of $\lambda_{t}$ in (5), it follows that its value is determined by $\lambda$ and future values of $\bar{u}_{k}^{*}, k>t+1$. Consequently, the model can be solved by sequentially solving Equation (4) for $t=T-1, \ldots, 0$, giving the parameters $\bar{u}_{t+1}^{*}$. An important property of the optimal solution is that (3) and (4) represent the solution to a static model $(T=1)$, with risk-aversion parameter $\lambda$ equal to $\lambda_{t}$. The multistage problem thus resembles a myopic model: it can be solved by solving a sequence of static problems. Only in this case the static problems are linked through the timevarying risk-aversion parameter $\lambda_{t}$.

The piecewise linearity of the optimal decision rule for $X_{t}$ in Theorem 3.1 holds for any set of absolute-continuous distribution functions $G_{t+1}(\cdot), t=0, \ldots, T-1$. $G_{t+1}(\cdot)$ only enters the optimal decision rule through the values of $\bar{u}_{t+1}^{+}$and $\bar{u}_{t+1}^{-}$, which follow easily from Equation (4). Theorem 3.1 thus provides the solution to the dynamic investment problem for standard assets such as a stock or bond index. Even if $G_{t+1}$ is the return distribution of a complicated derivative instrument, piecewise linearity of $X_{t}^{*}$ still holds.
Figure 2. Optimal investment in the risky asset.

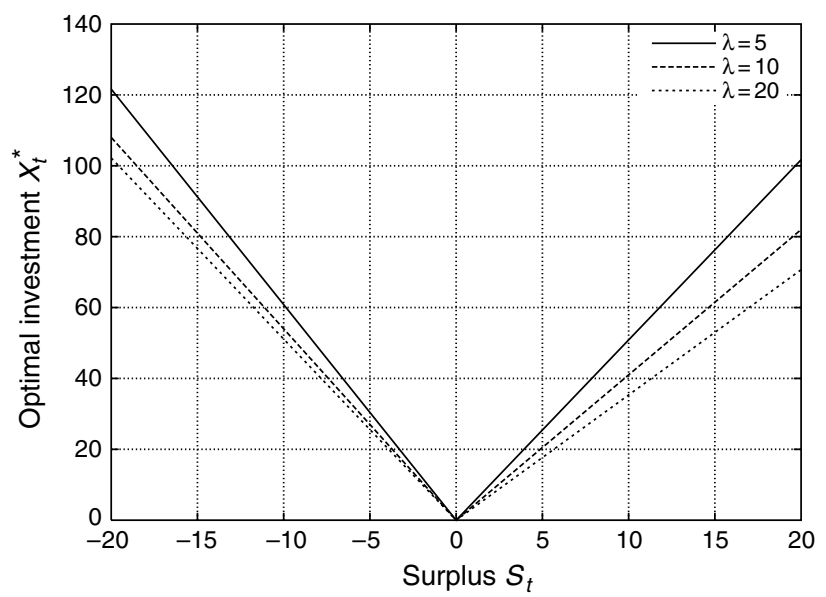

Notes. For a one-period model, this figure shows the optimal investment in the risky asset, $X_{0}^{*}$, as a function of initial surplus, $S_{0} \cdot r_{f}=1.04, u$ has a $\log$-normal distribution, with $\mu=0.085$, and $\sigma=0.16$, representing a typical stock return with a mean of $10 \%$ and standard deviation of $17 \%$.

\subsection{Risk Taking Under Shortfall}

For relevant parameter values and varying values of $\lambda$, Figure 2 plots the typical shape of the decision rule $X_{t}^{*}$. Risk taking is decreasing in the surplus when the surplus is negative, and increasing in the case it is positive. The optimality of a V-shaped portfolio policy is not confined to the specific mean-shortfall formulation. Similar effects were found based on a Kahneman-Tversky behavioral value function; see Berkelaar and Kouwenberg (2000a). Moreover, in $\S 4$ we show that the $\mathrm{V}$-shape persists for quadratic shortfall. The mean-shortfall objective and its solution thus explicitly characterize in a multistage setting the optimality of nonmonotonic investment policies that drive more general mean-downside-risk investment problems.

The shape of the investment policy for a positive surplus is intuitively clear. If wealth increases, more money can be invested in the risky asset at the cost of only a marginal increase in downside risk. This induces a positive relation between the surplus and the investment in the risky asset. Note that the results do not tend to risk-neutral allocations for large surplus values. Because $\bar{u}_{t}$ in (3) is fixed, the fraction invested in the risky asset tends to the constant $r^{f} /\left(r^{f}-\bar{u}_{t}^{*}\right)$ for large $S_{t}$. The outcome of a constant fraction is very similar to that of models with a constant relative risk-aversion (CRRA) utility function, such as power utility.

The most striking feature of Figure 2 is the increased risk taking if the surplus becomes increasingly negative. Such outcomes are generally normatively undesirable in practice. As the nonmonotic pattern of the investment policy appears to be robust for a variety of downside-risk preferences, we comment on it in more detail.

First, the decreasing left-hand side of the $\mathrm{V}$ shape does not disappear for $\lambda$ diverging to infinity, i.e., for large penalties on shortfall. Formally, this is clear from 
Theorem 3.1. Dividing the left-hand side and the right-hand side of Equation (4) by $\lambda_{t}$, the first-order condition for $\lambda \rightarrow \infty$ becomes

$\mathbb{E}_{t}\left[\left(r_{f}-u_{t+1}\right) \cdot I_{\left\{u_{t+1} \leqslant \bar{u}\right\}}\right]=0$.

The solution of (9) corresponds to the intersection of the dashed curve in Figure 1 with the horizontal axis. Given a finite value for $u_{t+1}^{*}$, risk taking will be nonzero. The intuitive explanation is that for large values of $\lambda$, the optimal solution under shortfall simplifies to the minimum-shortfall strategy. Given the assumption of a positive equity premium $\mathbb{E}[u]-r_{f}$, the investment in the risky asset converges to a nonzero value for $\lambda \rightarrow \infty$. Hence, the risk taking under shortfall (see, for example, Figure 1) is not due to considering only unrealistically low values for $\lambda$. Moreover, as mentioned earlier, the pattern is also robust to putting a convex rather than a linear penalty on shortfall; see $\$ 4$.

Second, it is important to realize that risk taking is not caused by convexity of the utility function in general, or convexity in losses (as in the Kahneman-Tversky specification), in particular. Instead, the result arises despite the explicit (and possibly large or increasing) penalty on losses.

One may argue that in practice institutional constraints mitigate the increased risk taking under shortfall, and thus the concerns of practitioners on the use of meandownside-risk optimization models. Such constraints can take the form of maxima on fractions invested in (classes of) risky assets, required stress tests, etc. Such institutional restrictions, however, obscure the key drivers of the basic models.

Another line of argument could be that increased risk taking under shortfall can be prevented by maximizing expected return subject to an explicit constraint on shortfall risk. If such constraints take the form of minimum return guarantees such that shortfall is prevented altogether, only the right-hand side of the $\mathrm{V}$-shaped policy becomes relevant. In many cases, however, such constraints are only of a probabilistic nature, such that there remains a nonzero probability of ending up in a negative surplus situation at some point in time. If such a situation happens to arise, the results in Theorem 3.1 make explicit the perhaps practically undesirable risk incentives induced by the use of a mean-downside-risk modeling framework.

Putting the results for positive and negative surplus together, our findings contrast with the general results for Hyperbolic Absolute Risk Aversion (HARA) utility functions. HARA utilities lead to a fraction invested in risky assets that is either monotonically increasing, decreasing, or constant in wealth. Here we find both decreasing and increasing behavior simultaneously.

\subsection{Risk Aversion and Value-at-Risk}

The model's solution yields a specific relation between probabilities of shortfall and the optimal investment in the risky asset. The following corollary gives an important property of the solution with respect to $\lambda$.
COROLlary 3.1. If the optimal solution is bounded and initial surplus is positive, then there is a unique $\lambda^{+}$such that under the optimal policies $X_{t}^{*}, t=0, \ldots, T-1, \operatorname{Pr}\left[W_{T}<\right.$ $\left.W^{B}\right]$ is fixed. For a negative initial surplus, there is a unique $\lambda^{-}$associated with the probability $\operatorname{Pr}\left[W_{T}>W^{B}\right]$.

Proof. See the appendix.

Corollary (3.1) states that a shortfall probability can be associated at the optimum with a unique value for the riskaversion parameter $\lambda$. Of course, the choices for the probabilities are limited by condition (8), which ensures that the optimal decisions $X_{t}^{*}$ are finite. For example, starting from a negative surplus the shortfall probability has a minimum value corresponding to the strategy that gives a positive final surplus with the highest probability, i.e., a minimumrisk strategy.

The financial industry widely uses the concept of valueat-risk ( $\mathrm{VaR})$, which is intimately related to the shortfall probabilities and benchmark wealth levels; see Jorion (2000). In a static context, Rockafellar and Uryasev (2000) propose a technique for stochastic portfolio optimization in which mean-shortfall (or CVaR) is optimized and VaR is calculated simultaneously. As such, Corollary 3.1 presents a similar result for the multistage optimization problem.

\subsection{Time Diversification}

Below we present two corollaries connecting our results in Theorem 3.1 to the phenomenon of time diversification. In essence, the proponents of time diversification claim that risky assets (e.g., stocks) become more attractive for longer investment horizons, because of the diversification of losses over time and the positive premium earned on holding risky assets. There is no consensus among academics, however, whether time diversification is fully in line with people's preferences, or whether it is an irrational or behavioral phenomenon. Using the multistage mean-downside-risk framework as our benchmark for loss averse preferences, we obtain the following results. The next section illustrates numerically how these results generalize toward a setting with quadratic shortfall.

Corollary 3.2. If (2) has a bounded solution and $T$ is fixed, the values of $\lambda_{t}$ are increasing in $t$.

Proof. See the appendix.

COROLlary 3.3. If the $u_{t}$ s are identically distributed, the absolute slope $r_{f} /\left|\bar{u}_{t+1}^{*}-r_{f}\right|$ of the optimal decision rules in (3) is monotonically increasing in the time to maturity $T-t$.

Proof. See the appendix.

As $\lambda_{t}$ represents the investor's risk aversion at time $t$, Corollary 3.2 states that in the multistage setting the investor becomes less risk averse the longer the time to maturity $T-t$. The effect of $\lambda_{t}$ on the investment policy $X_{t}^{*}$ is illustrated in Figure 2. As a direct result, Corollary 3.3 
concludes that the optimal policies are more sensitive to changes in surplus for longer planning horizons. Moreover, the increasing slopes of the decision rules also imply a higher level of risky investments for longer planning periods and given initial surplus.

The intuition given by proponents of time diversification for the decrease in risk aversion for longer planning horizons is straighforward: in the long run the increased uncertainty due to a more risky portfolio is more than compensated by the higher expected return. Some base this view on the obvious observation that the probability of a lower than risk-free return decreases with a longer horizon. However, Samuelson (1994), Kritzman and Rich (1998), and Merton and Samuelson (1974) argue that the spread of the distribution of terminal wealth also widens with the time horizon. Consequently, whereas the probability of a loss decreases, the potential magnitude of the loss increases accordingly. This is used as an argument to claim that investors might well choose less aggressive portfolios if their investment horizon increases. Corollary 3.3 highlights that if wealth is measured against a benchmark level and the magnitude of a loss is (linearly) taken into account, time diversification holds for any distribution of the risky returns with a positive risk premium. Hence, the mean-shortfall framework, in fact, supports a preference-based explanation of time diversification. The basic driver of this result appears to be the fact that the model allows for a situation of shortfall. As explained earlier, a larger shortfall results in a higher investment in the risky asset. This resembles a doubling strategy, but with a predetermined number of bets (equal to the number of remaining decision moments). If the number of bets increases, it is therefore not surprising that the asset allocation for a given level of surplus also becomes riskier.

\subsection{Trade Frequency}

As mentioned in $\$ 2$, one of the drawbacks of the mean shortfall model is the local risk neutrality of the objective function. In a complete markets setting, this results in undesirable betting behavior, also labeled casino behavior by Dert and Oldenkamp (2000). Such behavior can only be controlled by a more complete specification of preferences or by imposing additional restrictions on the planning problem.

Our model is set in an incomplete markets framework. Still, however, the casino effect is important. This is easily seen if we increase the number of stages, i.e., the trade frequency for a given length of the planning period. This effectively amounts to making the market dynamically complete, thus giving rise to similar degenerate solutions as in Dert and Oldenkamp (2000). This is illustrated in Figure 3. For simplicity, we assume that risky return prices follow a log-Brownian motion with drift on the unit interval. The left-hand panel in Figure 3 shows the minimum risk-aversion parameter $\lambda$ that satisfies the "finite-solution" constraint (8) at $t=0$. As expected, the risk-aversion parameter has to increase (more than) exponentially in the number of decision moments to retain a finite solution. The right-hand panel in Figure 3 shows a similar effect. For a given risk-aversion parameter, it shows the amount of risky investments (on a log scale) as a function of the number of decision moments in the $[0,1]$ interval. It is clear that the amount increases more than exponentially in the number of decision moments.

The results illustrate again one of the problems with empirical financial planning models based on expected returns and downside-risk measures. As shown, in a multistage setting, the key drivers of such models are geared towards casino-type solutions. The fact that such solutions are not immediately apparent may be due to the fact that institutional constraints obscure the basic mechanisms of such models. For a more elaborate dynamic (decision) specification of the model, however, including more decision moments, a more complete and nonlinear specification of preferences is needed. In the continuous time limit, strict risk aversion is needed to ensure nondegenerate solutions; see Basak and Shapiro (2001) and Berkelaar and Kouwenberg (2000a). A similar caveat may apply to many

Figure 3. Threshold values for $\lambda$ and risky investment for increasing number of stages.
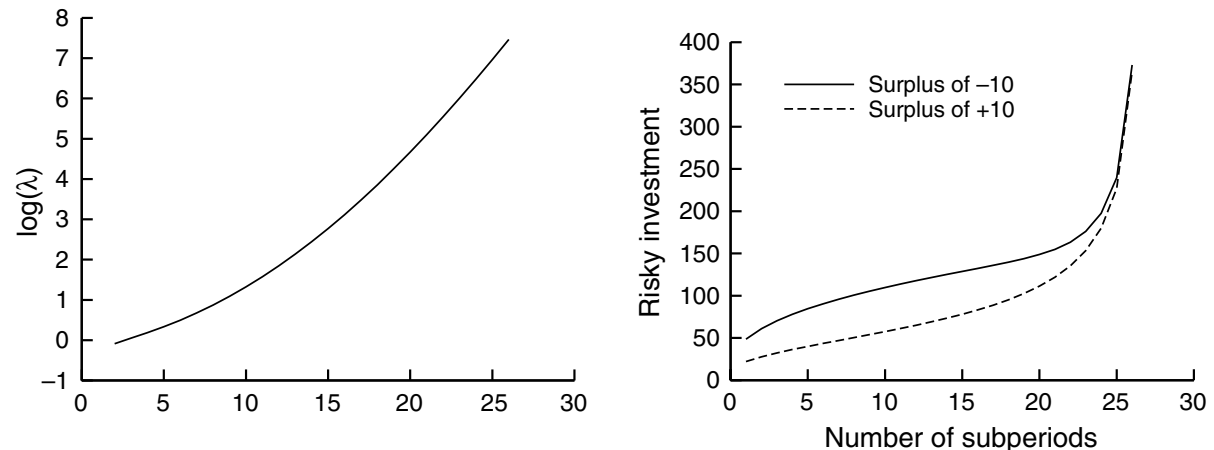

Notes. The left-hand panel shows the threshold value for $\lambda$ that gives a bounded solution as a function of the number of subperiods. The returns are assumed $\log$-normal $(0.085,0.16)$. For a given value of $\lambda$ equal to $\exp (8.5)$, the right-hand panel features the investment in the risky asset for a surplus of plus and minus 10 , respectively, and increasing subperiods. 
of the financial planning models applied in practice that fail to account for risk aversion in upside potential.

\section{Quadratic Shortfall}

So far, the analysis was based on a downside-risk measure that was linear in shortfall. In this section, we analyze a downside-risk measure that is strictly concave in losses, i.e., that puts an increasing marginal penalty on losses. Although this specification is less tractable than the meanshortfall model, we are able to corroborate the results and conclusions from the previous section. We derive analytical results for the one-period model under quadratic shortfall, and numerical results on time diversification for the multistage model.

Risk measures that put strictly convex penalties on losses may be more desirable from a normative financial planning point of view. Using a risk measure that is concave in losses is recommended in, for example, Sortino and Van der Meer (1991) and Harlow (1991). Specifically, they argue that downside deviation (or semi-deviation, as it is called by Markowitz 1959), is the risk measure to be used in investment. See also Boender (1997) and in the practice of ALM, see Ziemba and Mulvey (1998).

Our aim is to determine if and how the convex, nonmonotonic asset allocation policies as a function of the surplus persist if the risk measure becomes quadratic in shortfall. To do so, we analyze a one-period investment model as before, but now with an objective function that penalizes squared shortfall below a benchmark level of wealth. With $T=1$, the formulation of the objective is

$\max _{X_{0}} \mathbb{E}\left[W_{1}\right]-\nu \cdot \mathbb{E}\left[\left(\left(W^{B}-W_{1}\right)^{+}\right)^{2}\right]$,

where the variables are the same as in the previous section. The only exception is the new variable $\nu$, representing loss aversion with respect to the squared shortfall below $W^{B}$.

The main results for the model with a squared shortfall objective are in the following theorem.

THEOREM 4.1. For $\nu>0$, the optimal solution $X_{0}^{*}$ to problem (10) has the following properties:

(i) The optimal stock investment $X_{0}^{*}$ for zero surplus $\left(S_{0}=0\right)$ is positive, decreasing in $\nu$, and given by

$X_{0}^{*}=\frac{\mathbb{E}\left[u-r_{f}\right]}{2 \nu \cdot \mathbb{E}\left[\left(r_{f}-u\right)^{+}\right]^{2}}$.

(ii) The optimal decision $X_{0}^{*}$ as a function of $S_{0}$ has one minimum for a nonpositive surplus. For any $\nu$, the minimum lies on the straight line characterized by

$X_{0}=\frac{r_{f}}{r_{f}-\bar{u}^{m}} S_{0}$,

where $\bar{u}^{m}$ is the $\bar{u}>r_{f}$ that solves

$\int_{0}^{\bar{u}}\left(u-r_{f}\right) d G=0$. (iii) For a positive surplus, and $\nu \rightarrow \infty, X_{0}^{*}=S_{0}$. For a negative surplus, and $\nu \rightarrow \infty, X_{0}^{*}$ is given by

$X_{0}^{*}=\frac{r_{f}}{r_{f}-\bar{u}^{*}} S_{0}$,

where $\bar{u}^{*}$ is the $\bar{u}>r_{f}$ that solves

$\int_{0}^{\bar{u}}\left(r_{f}-u\right) d G=\frac{1}{r_{f}-\bar{u}} \int_{0}^{\bar{u}}\left(u-r_{f}\right)^{2} d G$.

Moreover, for any given negative surplus, and $\nu \rightarrow \infty, X_{0}^{*}$ is smaller than for the mean-shortfall objective with $\lambda \rightarrow \infty$.

Proof. See the appendix.

Theorem 4.1 gives specific properties of the solution, without completely specifying a parametric decision rule for the optimal $X_{0}^{*}$ as a function of $S_{0}$. We now discuss the results and compare them to the results in Theorem 3.1. In contrast to the mean-shortfall model, the existence of a bounded solution does not depend on a lower bound for the risk-aversion parameter $\nu$. In the mean-shortfall model, the marginal value of gains and losses is fixed. When the objective is quadratic in losses and linear in gains, there always exists an allocation where the marginal penalty on losses exceeds the marginal value of gains.

Theorem 4.1(i) shows that the optimal investment in the risky asset is nonzero at zero surplus. This is a direct result of the quadratic penalty function $\left[\left(W^{B}-W_{1}\right)^{+}\right]^{2}$, which has zero slope at $W_{1}=W^{B}$. This is different from the meanshortfall objective, where the marginal penalty is constant and nonzero regardless of the distance to the benchmark. A large value of $\nu$, however, drives $X_{0}^{*}$ at $W_{0}=W_{0}^{B}$ to zero. In many cases, it will be undesirable from a normative point of view to allow for positive investments in the risky asset if the surplus is zero. This is especially concerning given the use of quadratic downside-risk measures in empirical financial planning models; see e.g. Boender (1997).

Second, the implication of (ii) is that the optimal investment in the risky asset has a nonmonotonic convex relation with the surplus $S_{0}$. For different values of $\nu$, the minimum stock investments lie on a straight line through the point with $S_{0}=X_{0}^{*}=0$. Equation (13) resembles the first-order condition for the mean-shortfall model in Equation (4) with $\lambda_{t} \rightarrow \infty$. Given that for the mean-shortfall model $X_{0}=0$ for $S_{0}=0$, and given the nonzero minimum investment $X_{0}^{\min }$ for the quadratic-shortfall case, the implication is that for each value of $\nu$ and $\lambda$ there is an area around $S_{0}=0$, where the investment in the risky asset is higher in the squared-shortfall model than in the linear-shortfall model. So despite the concavity in losses, there is a range of initial negative and positive surplus values for which the meanquadratic-shortfall objective results in riskier allocations than the mean-linear-shortfall objective.

The limiting behavior for $X_{0}^{*}$ when $\nu \rightarrow \infty$ in (iii) shows that for large values of $\nu$, the relation between the risky investment and surplus becomes piecewise linear again. 
For positive surplus, the optimal $X_{0}^{*}$ is equal to the surplus itself, as the aversion to downside risk has become too large. For negative surplus, however, the solution converges to an allocation that gives minimum quadratic shortfall. Although smaller than in the linear-shortfall case, the investment in the risky asset does not go to zero for negative surplus. Ex ante intuition might say that high loss aversion induces low-risk allocations. This is clearly not true if one starts in a situation of shortfall. For the surplus going to plus or minus infinity, the optimal decision also becomes a V-shaped function of the surplus; see the proof in the appendix. This suggests that the solution to the quadraticshortfall case can be approximated by a piecewise linear decision rule.

Figure 4 shows the numerical solutions to model (10) given three different values of $\nu$. It illustrates the results in Theorem 4.1, and also shows the typical shapes of the optimal decision rules, together with an idea of the sensitivity of the optimal solution to $\nu$.

We see in Figure 4 that the the right-hand side of the curves is more sensitive to changes in $\nu$ than the left-hand side. For $\nu=10$, the curve becomes virtually piecewise linear as suggested by part (iii) of the theorem.

\subsection{Time Diversification}

Having established the results on the optimal decisions under squared shortfall for a one-period model, we now turn to the robustness of our results on time diversification. In the case of expected shortfall, we used the special structure of the objective to solve the multistage model explicitly. This is not possible for the quadratic-shortfall model. Therefore, we solve a two-period model numerically and

Figure 4. Solution for the quadratic-loss objective.

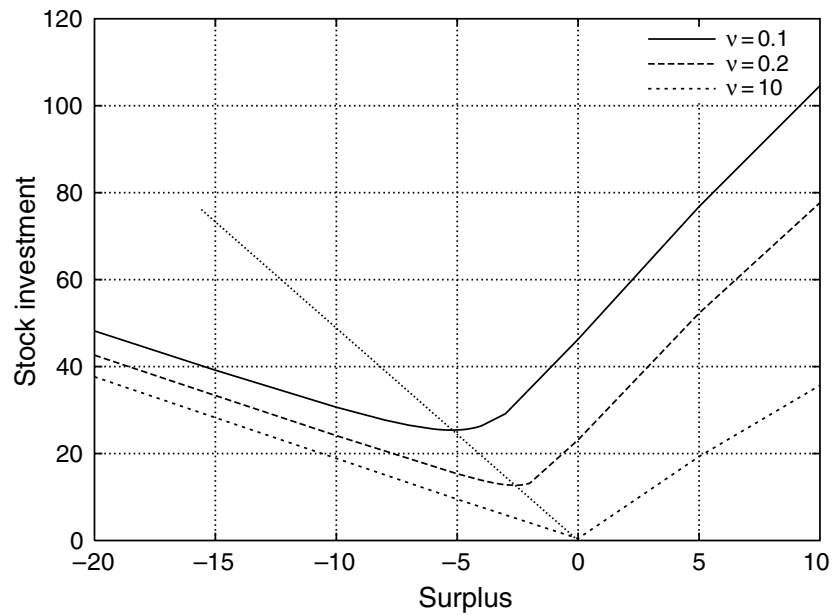

Notes. For a one-period model, this figure shows the optimal investment in the risky asset as a function of initial surplus. The objective is to maximize expected return minus $\nu$ times quadratic shortfall below a benchmark asset level $W^{B}$. The figure displays results for $W^{B}=104, r_{f}=1.04$, and $u \sim \log$-normal $(0.085,0.16)$. The added line goes through the minima of the decision rules.
Figure 5. Multiple periods in the quadratic-loss model.

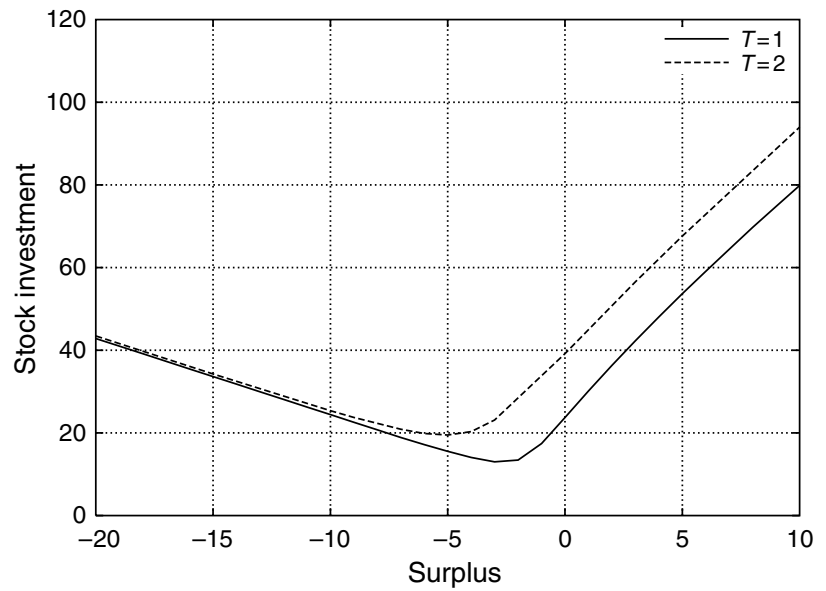

Notes. This figure shows the optimal initial investment in the risky asset as a function of initial surplus for the quadratic-loss objective with a horizon of one and two periods. Benchmark $W^{B}$ is 104 for $T=1$ and 108.16 for $T=2 . r_{f}=1.04$, and $u_{t} \sim \log$-normal $(0.085,0.16)$. The solution is computed by discretizing the distribution of $u_{t}$ using 30 evenly spaced points.

study the effect on our results for time diversification. Figure 5 shows the solutions to the one- and two-period models with squared shortfall as the risk measure.

We observe that the general result for the mean-shortfall model in Corollary 3.3 continues to hold. For a given time- $t$ surplus, the allocation to the risky asset is larger for the two-period model than for the one-period model. Also, the bottom of the U-shaped decision rule is shifted to the upper left, comparable to the effect of a decreasing $\nu$. Note that due to the convexity of the penalty on losses, the timediversification effect for a (large) negative initial surplus is very limited. This constitutes an important difference with the case of linear shortfall; see also Figure 2 and Corollary 3.2. Numerically solving a three-period model produced computational problems for acceptable sizes of the discretizations. As the number of periods grow, the scenario tree and hence the problem size increases exponentially. See also Berkelaar et al. (2000, 2002), who introduce state-of-the-art interior point methods to solve multistage stochastic programming models, but still only solve problems for scenario trees of very limited size.

\section{Conclusions and Discussion}

In this paper, we analytically characterized the solution to a multistage financial planning model involving a tradeoff between return and downside risk. Our model shares the basic characteristics of more elaborate empirical models such as that of Cariño et al. (1994). Downside risk was used because of its widespread popularity in the financial industry and its use in related academic work. Risk was measured with respect to a benchmark wealth level. This can be interpreted as a liabilitity level in the case of asset/liability management (ALM) problems. It is also the most realistic form 
of risk measurement emerging from behavioral research; see Kahneman and Tversky (1979). We have derived the analytic characterization of the optimal solutions in feedback form, i.e., as decision rules. This form has a clear-cut economic interpretation, which constitutes a valuable addition to the existing literature where solutions are often derived numerically rather than analytically.

One of the salient findings of this paper is that the optimal decision rule has a V- or U-shaped relation with the surplus for either a linear or quadratic penalty on losses. Moreover, the results do not hinge on the specific probability distribution of the return on the risky asset. The distribution only determines the relative steepness of the decision rule for positive and negative values of the surplus. Numerical results in Siegmann (2003) further show that the V-shaped rules persist for restrictions on allocations, a stochastic benchmark, and a loss-averse utility function directly taken from Tversky and Kahneman (1992). See also the results in Berkelaar and Kouwenberg (2000a, 2000 b), who solve asset allocation problems in continuoustime models with log-normal distributed returns and a general specification of loss aversion.

\section{Appendix. Proofs}

Proof of Theorem 3.1. We solve the optimization problem as given by Equations (1) and (2) by dynamic programming, and begin with the following sequence of value functions:

$$
\begin{aligned}
& V_{t}\left(W_{t}\right)=\max _{X_{t}} \mathbb{E}_{t}\left[V_{t+1}\left(W_{t} r_{f}+X_{t}\left(u_{t+1}-r_{f}\right)\right)\right], \\
& \qquad t=0, \ldots, T-1, \\
& V_{T}\left(W_{T}\right)=W_{T}-\lambda \cdot\left[W_{T}^{B}-W_{T}\right]^{+},
\end{aligned}
$$

where $\mathbb{E}_{t}$ is defined as the conditional expectation given $u_{t-1}, u_{t-2}, \ldots, u_{1}$. Given $W_{t}, V_{t}\left(W_{t}\right)$ is the expected value of the objective function when all decisions from time $t$ on are taken optimally. Clearly, solving the model is equivalent to finding $V_{0}\left(W_{0}\right)$.

The first-order condition with respect to $X_{T-1}$ is obtained by differentiating $V_{T}\left(W_{T}\right)$ to $X_{T-1}$ and is given by

$\mathbb{E}_{T-1}\left[u_{T}-r_{f}\right]+\lambda \cdot \int_{0}^{\bar{u}_{T}}\left(u_{T}-r_{f}\right) d G_{T}\left(u_{T}\right)=0$,

where $\bar{u}_{T}$ is defined as

$\bar{u}_{T}=r_{f}+\frac{W_{T}^{B}-W_{T-1} \cdot r_{f}}{X_{T-1}}$.

As shown in the main text, if condition (8) holds, (A3) solves for two distinct values of $\bar{u}_{T}$. To prove that the resulting $X_{T-1}^{*}$ is optimal, we consider the second-order condition for the problem at $T-1$ :

$\frac{\partial^{2} V_{T}\left(W_{T}\right)}{\partial X_{T-1}^{2}}=-\lambda \cdot \frac{\left(W_{T}^{B}-W_{T-1} r_{f}\right)^{2}}{X_{T-1}^{3}} \cdot g_{T}\left(\bar{u}\left(X_{T-1}\right)\right)<0$, where $g_{t}(\cdot)$ is defined as the density function of $u_{t}$ for $t=1, \ldots, T$.

As $\lambda>0$, it follows from (A5) that an optimal $X_{T-1}^{*}$ should be positive at the optimum. Therefore, only a $\bar{u}_{T}<$ $r_{f}$ can be optimal if $W_{T}^{B}-W_{T-1} r_{f}<0$. Otherwise, $X_{T-1}^{*}$ would be negative. Also, only a $\bar{u}_{T}>r_{f}$ can be optimal if $W_{T}^{B}-W_{T-1} r_{f}>0$. As these two cases correspond to a positive and a negative surplus, respectively, we define $\bar{u}_{T}^{+}$ and $\bar{u}_{T}^{-}$as the corresponding values of $\bar{u}$.

As $X_{t-1}$ influences the objective function only through the effect on $W_{t}$, i.e., there are no direct costs associated with a choice of $X_{t-1}$, the first-order condition with respect to $X_{t-1}, t<T$ is

$\mathbb{E}_{t-1}\left[\frac{\partial V_{t}\left(W_{t}\right)}{\partial X_{t-1}}\right]=\mathbb{E}_{t-1}\left[\frac{\partial V_{t}\left(W_{t}\right)}{\partial W_{t}} \cdot \frac{\partial W_{t}}{\partial X_{t-1}}\right]=0$.

Let $W_{t+1}^{*}=r_{f} W_{t}+X_{t}^{*} \cdot\left(u_{t+1}-r_{f}\right)$, with $X_{t}^{*}$ the optimal decision at time $t$. This implies that $\partial W_{t+1} / \partial W_{t}=r_{f}$, such that

$$
\begin{aligned}
\frac{\partial V_{t}\left(W_{t}\right)}{\partial W_{t}} & =\mathbb{E}_{t}\left[\frac{\partial V_{t+1}\left(W_{t+1}\right)}{\partial W_{t+1}} \cdot \frac{\partial W_{t+1}^{*}}{\partial W_{t}}\right] \\
& =r_{f} \cdot \mathbb{E}_{t}\left[\frac{\partial V_{t+1}\left(W_{t+1}\right)}{\partial W_{t+1}}\right]=r_{f}^{2} \cdot \mathbb{E}_{t}\left[\frac{\partial V_{t+2}\left(W_{t+2}\right)}{\partial W_{t+2}}\right] \\
& =\cdots=r_{f}^{T-t} \mathbb{E}_{t}\left[1+\lambda \cdot I_{\left\{S_{T}<0\right\}}\right],
\end{aligned}
$$

where $S_{t}$ is defined as the surplus $W_{t}-W_{t}^{B}$ at time $t$, and $I_{A}$ is the indicator function of the event $A$. In this case, it is equal to 1 if $S_{T}<0$ and 0 otherwise.

Using (A7) and the fact that $\partial W_{t} / \partial X_{t-1}=u_{t}-r_{f}$, the first-order condition in (A6) becomes

$$
\begin{aligned}
\mathbb{E}_{t-1}\left[\frac{\partial V_{t}\left(W_{t}\right)}{\partial X_{t-1}}\right]= & \mathbb{E}_{t-1}\left[r_{f}^{T-t} \cdot\left(u_{t}-r_{f}\right)\right] \\
& +\lambda \cdot \mathbb{E}_{t-1}\left[r_{f}^{T-t} \cdot\left(u_{t}-r_{f}\right) \cdot I_{\left\{S_{T}<0\right\}}\right]=0 .
\end{aligned}
$$

Dividing by $r_{f}^{T-t}$ and partitioning based on the sign of $S_{t}$ gives

$$
\begin{aligned}
& \mathbb{E}_{t-1}\left[u_{t}-r_{f}\right]+\lambda \cdot \mathbb{E}_{t-1}\left[\left(u_{t}-r_{f}\right) \cdot I_{\left\{S_{T}<0\right\} \cap\left\{S_{t}<0\right\}}\right] \\
& +\lambda \cdot \mathbb{E}_{t-1}\left[\left(u_{t}-r_{f}\right) \cdot I_{\left\{S_{T}<0\right\} \cap\left\{S_{t}>0\right\}}\right]=0 .
\end{aligned}
$$

Note that for given $W_{t-1}, X_{t-1}^{*}$ is fixed. As the $u_{t}$ s are independent, $S_{t}$ only varies monotonically with the realization of $u_{t}$. By absolute continuity of $G_{t}(\cdot)$, we do not have to consider the case $S_{t}=0$, so we can define a $\bar{u}_{t}$ such that $S_{t}>0$ for $u_{t}>\bar{u}_{t}$ and $S_{t}<0$ for $u_{t}<\bar{u}_{t}$. This reduces (A9) to

$$
\begin{aligned}
& \mathbb{E}_{t-1}\left[u_{t}-r_{f}\right]+\lambda \cdot \int_{0}^{\bar{u}_{t}}\left(u_{t}-r_{f}\right) \operatorname{Pr}\left(S_{T}<0 \mid S_{t}\right) d G_{t}\left(u_{t}\right) \\
& \quad+\lambda \cdot \int_{\bar{u}_{t}}^{\infty}\left(u_{t}-r_{f}\right) \operatorname{Pr}\left(S_{T}<0 \mid S_{t}\right) d G_{t}\left(u_{t}\right)=0
\end{aligned}
$$


Assume that

$\operatorname{Pr}\left(S_{T}<0 \mid S_{t}\right)= \begin{cases}p_{t}^{+} & \text {if } S_{t}>0, \\ p_{t}^{-} & \text {if } S_{t}<0 .\end{cases}$

The above assumption states that the probability of ending up with a negative terminal surplus only depends on the sign of the time $t$ surplus $S_{t}$ and not on its value. This clearly holds for $t=T$ with $p_{T}^{+}=0$ and $p_{T}^{-}=1$. Equation (A11) implies that Equation (A10) can be rewritten as

$$
\begin{gathered}
\mathbb{E}_{t-1}\left[u_{t}-r_{f}\right]+\lambda \cdot p_{t}^{-} \cdot \int_{0}^{\bar{u}_{t}}\left(u_{t}-r_{f}\right) d G_{t}\left(u_{t}\right)+\lambda \cdot p_{t}^{+} \\
\cdot \int_{\bar{u}_{t}}^{\infty}\left(u_{t}-r_{f}\right) d G_{t}\left(u_{t}\right)=0,
\end{gathered}
$$

which simplifies to

$$
\begin{gathered}
\left(1+\lambda \cdot p_{t}^{+}\right) \cdot \mathbb{E}_{t-1}\left[u_{t}-r_{f}\right]+\lambda \cdot\left(p_{t}^{-}-p_{t}^{+}\right) \\
\cdot \int_{0}^{\bar{u}_{t}}\left(u_{t}-r_{f}\right) d G_{t}=0,
\end{gathered}
$$

or, dividing by $\left(1+\lambda \cdot p_{t}^{+}\right)$,

$\mathbb{E}_{t-1}\left[u_{t}-r_{f}\right]+\frac{\lambda \cdot\left(p_{t}^{-}-p_{t}^{+}\right)}{\left(1+\lambda \cdot p_{t}^{+}\right)} \cdot \int_{0}^{\bar{u}_{t}}\left(u_{t}-r_{f}\right) d G_{t}=0$.

Defining $\lambda_{t-1}$ as

$\lambda_{t-1}=\frac{\lambda \cdot\left(p_{t}^{-}-p_{t}^{+}\right)}{1+\lambda \cdot p_{t}^{+}}$,

we can write (A14) as

$\mathbb{E}_{t-1}\left[u_{t}-r_{f}\right]+\lambda_{t-1} \cdot \int_{0}^{\bar{u}_{t}}\left(u_{t}-r_{f}\right) d G_{t}=0$.

As Equation (A16) is the time- $t$ version of the first-order condition for the problem at $T-1$, we can use the exact same reasoning as for Equation (A3). So, if condition (8) holds, this equation solves for two distinct $\bar{u}_{t}$, corresponding to a positive and a negative surplus, respectively. Moreover, the corresponding optimal $X_{t-1}^{*}$ is such that the next period's surplus, $S_{t}$, is positive for a realization of the risky return $u_{t}>\bar{u}_{t}$ and negative for $u_{t}<\bar{u}_{t}$. Therefore,

$$
\begin{aligned}
\operatorname{Pr}\left(S_{T}<0 \mid S_{t-1}\right) & \\
= & \operatorname{Pr}\left(S_{T}<0 \mid S_{t}<0\right) \cdot \operatorname{Pr}\left(S_{t}<0 \mid S_{t-1}\right) \\
& \quad+\operatorname{Pr}\left(S_{T}<0 \mid S_{t}>0\right) \cdot \operatorname{Pr}\left(S_{t}>0 \mid S_{t-1}\right) \\
= & p_{t}^{-} \cdot \int_{0}^{\bar{u}_{t}} d G_{t}\left(u_{t}\right)+p_{t}^{+} \cdot \int_{\bar{u}_{t}}^{\infty} d G_{t}\left(u_{t}\right),
\end{aligned}
$$

where $\bar{u}_{t}=\bar{u}_{t}^{+}$for $S_{t-1}>0$ and $\bar{u}_{t}=\bar{u}_{t}^{-}$for $S_{t-1}<0$. As both $\bar{u}_{t}^{+}$and $\bar{u}_{t}^{-}$are constant, (A18) clearly shows that $\operatorname{Pr}\left(S_{T}<0 \mid S_{t-1}\right)$ also satisfies (A11). The proof now follows by induction.

Proof of Corollary 3.1. Define $G_{t}^{-}$and $G_{t}^{+}$by $G_{t}\left(\bar{u}_{t}^{-}\right)$ and $G_{t}\left(\bar{u}_{t}^{+}\right)$, respectively. Given a value of $\lambda, \bar{u}_{T}^{+}$and $\bar{u}_{T}^{-}$are uniquely determined for the decision problem at time $T-1$. In turn, $\bar{u}_{T}^{+}$and $\bar{u}_{T}^{-}$determine $G_{T}^{+}$and $G_{T}^{-}$which determine $p_{T-1}^{+}$and $p_{T-1}^{-}$. Repeating this procedure for all previous subproblems finally gives a unique $p_{0}^{+}$or $p_{0}^{-}$, depending on whether $S_{0}>0$ or $S_{0}<0$, respectively. This implies that if a certain $p_{0}^{+}$or $p_{0}^{-}$is chosen, there is either an unbounded solution, or a bounded solution with a unique $\lambda$ associated with it.

Proof of Corollary 3.2. The risk-aversion parameter at time $t, \lambda_{t}$ follows from (A15). From (A18) we have that $p_{t}^{+}$and $p_{t}^{-}$are defined recursively as

$p_{t}^{-}=p_{t+1}^{-} \cdot G_{t}\left(\bar{u}_{t}^{-}\right)+p_{t+1}^{+}\left(1-G_{t}\left(\bar{u}_{t}^{-}\right)\right)$,

$p_{t}^{+}=p_{t+1}^{-} \cdot G_{t}\left(\bar{u}_{t}^{+}\right)+p_{t+1}^{+}\left(1-G_{t}\left(\bar{u}_{t}^{+}\right)\right)$,

and $p_{T}^{-}=1, p_{T}^{+}=0$.

As $\bar{u}_{t}^{-}>\bar{u}_{t}^{+}$, we have $1>G_{t}^{-}>G_{t}^{+}>0$ for any $t$. Using (A19) and (A20), we can write

$p_{t}^{-}-p_{t}^{+}=\left(p_{t+1}^{-}-p_{t+1}^{+}\right) \cdot\left(G_{t+1}^{-}-G_{t+1}^{+}\right), \quad i=0, \ldots, n-1$.

As $p_{T}^{-}>p_{T}^{+}$and $\left(G_{t+1}^{-}-G_{t+1}^{+}\right)<1$ for all $t$, this implies that

$p_{t}^{-}-p_{t}^{+}<p_{t+1}^{-}-p_{t+1}^{+}, \quad t=0, \ldots, T-1$,

i.e., $\left\{p_{t}^{-}-p_{t}^{+}\right\}_{0}^{T}$ is a strictly positive and increasing sequence in $t$. Moreover, as $p_{t}^{-}>p_{t}^{+}$, it follows from (A20) that $p_{t+1}^{+}<p_{t}^{+}$. Using this and (A22), it is easy to see that the numerator in the definition of $\lambda_{t-1}$ (A15) is increasing in $t$, while the denominator is decreasing in $t$. Hence, $\lambda_{t}$ is increasing in $t$, and thus decreasing in the time to maturity $T-t$.

Proof of Corollary 3.3. With identically distributed returns $u_{t}, t=1, \ldots, T$, the solution to (4) depends only on the value of $\lambda_{t}$. As can be observed from Figure 1, a larger value of $\lambda_{t}$ corresponds to a larger distance between the $\bar{u}_{t+1}$ s and $r_{f}$. This implies that the distance $\left|r_{f}-\bar{u}_{t+1}^{*}\right|$ is increasing in $t$. Hence, the absolute slope $r_{f} /\left|r_{f}-\bar{u}_{t}^{*}\right|$ in the decision rule for $X_{t}^{*}$ is decreasing in $t$.

Proof of Theorem 4.1. The maximization problem to solve is

$\max _{X_{0}} \mathbb{E}\left[W_{1}\right]-\nu \mathbb{E}\left[\left(\left\{W^{B}-W_{1}\right\}^{+}\right)^{2}\right]$,

where $W_{1}=W_{0} r_{f}+X_{0} \cdot\left(u-r_{f}\right)$. The first-order condition to this problem can be written as

$$
\begin{array}{r}
\mathbb{E}\left[u-r_{f}\right]-2 \nu r_{f} S_{0} \int_{0}^{\bar{u}}\left(u-r_{f}\right) d G \\
-2 \nu X_{0} \int_{0}^{\bar{u}}\left(u-r_{f}\right)^{2} d G=0,
\end{array}
$$


where $S_{0}=W_{0}-W^{B} / r_{f}$, and $\bar{u}$ is defined as

$\bar{u}=-\frac{S_{0} r_{f}}{X_{0}}+r_{f}$,

giving the return for which $W_{1}=W^{B}$. The second-order condition is given by

$-2 \nu \int_{0}^{\bar{u}}\left(u-r_{f}\right)^{2} d G<0$,

which is satisfied for $\nu>0$.

We prove the three parts of the theorem separately.

(i) If $S_{0}=0, \bar{u}=r_{f}$, so Equation (A24) simplifies to

$\mathbb{E}\left[u-r_{f}\right]-2 \nu X_{0} \int_{0}^{r_{f}}\left(u-r_{f}\right)^{2} d G=0$.

The left-hand side of (A27) is a linear equation in $X_{0}$, with solution

$X_{0}^{*}=\frac{\mathbb{E}\left[u-r_{f}\right]}{2 \nu \mathbb{E}\left[\left(r_{f}-u\right)^{+}\right]^{2}}$,

which establishes that $X_{0}^{*}$ at zero surplus is positive and decreasing in $\nu$.

(ii) Given the first-order condition (A24) and using the implicit function theorem, the derivative of $X_{0}^{*}$ with respect to $S_{0}$ is given by

$\frac{\partial X_{0}^{*}}{\partial S_{0}}=-\frac{\partial F / \partial S_{0}}{\partial F / \partial X_{0}}$,

where we have used $F(\cdot)$ to denote the derivative of the objective function with respect to $X_{0}$, being the left-hand side of (A24). From the second-order condition in (A26), we know that the denominator of (A29) is negative. The numerator is given by

$\frac{\partial F}{\partial S_{0}}=-2 \nu r_{f} \int_{0}^{\bar{u}}\left(u-r_{f}\right) d G$,

which is zero for $\bar{u}=0$ and some $\bar{u}^{m}>r_{f}$. We can ignore the case $\bar{u}=0$, as $\bar{u} \downarrow 0$ is only a solution to the first-order condition (A24) if $\nu \rightarrow \infty$ or $\left|S_{0}\right| \rightarrow \infty$. From the definition of $\bar{u}$ in (A25) and the fact that $\bar{u}^{m}>r_{f}$, it follows that the surplus at which $X_{0}^{*}=X_{0}^{\min }$ lies at a negative surplus. Combining (A24) with (A30), an alternative expression for $X_{0}^{\min }$ is given by

$X_{0}^{\min }=\frac{\mathbb{E}\left[u-r_{f}\right]}{2 \nu \int_{0}^{\bar{u}^{m}}\left(u-r_{f}\right)^{2} d G}$.

From the second-order derivative $\partial^{2} F / \partial S_{0}^{2}$, which is positive, we find that $X_{0}^{\min }$ is the minimum investment in the risky asset. With respect to the sign of $X_{0}^{*}$, note that from (i) we have $X_{0}^{*}\left(S_{0}=0\right)>0$. Because we have that $\partial X_{0}^{*} / \partial S_{0}$ is a continuous function of $S_{0}$, and $X_{0}=0$ does not solve the first-order condition (A24), we find that $X_{0}^{*}$ is positive.
Figure 6. Solutions to Equation (A33).

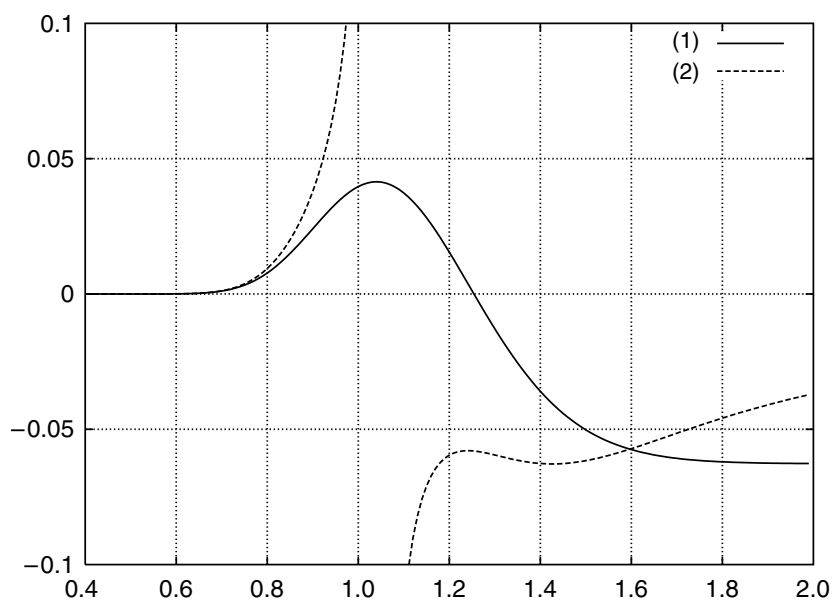

Notes. This figure shows the left-hand (1) and right-hand (2) sides of Equation (A33) as a function of $\bar{u} . r_{f}=1.04$ and $u \sim \log N(0.085,0.16)$.

(iii) Dividing the left-hand side expression of the firstorder condition (A24) by $2 \nu S_{0} r_{f}$ gives

$$
\begin{aligned}
& \frac{\mathbb{E}\left[u-r_{f}\right]}{2 r_{f} \nu S_{0}}-\int_{0}^{\bar{u}}\left(u-r_{f}\right) d G \\
& \quad+\frac{1}{\bar{u}-r_{f}} \int_{0}^{\bar{u}}\left(u-r_{f}\right)^{2} d G=0 .
\end{aligned}
$$

For $S_{0} \neq 0$ and $\nu \rightarrow \infty$, the expression in (A32) simplifies to

$\int_{0}^{\bar{u}}\left(r_{f}-u\right) d G=\frac{1}{r_{f}-\bar{u}} \int_{0}^{\bar{u}}\left(u-r_{f}\right)^{2} d G$,

which depends on $r_{f}$ and $G(\cdot)$ only. As (A33) does not include terms $W_{0}$ or $X_{0}$, we find that in the limit, $X_{0}^{*}$ is determined by the $\bar{u}$ s that solve (A33). For given parameter values, Figure 6 shows the left- and right-hand sides of Equation (A33), as a function of $\bar{u}$. There is a clear point of intersection for $\bar{u}>r_{f}$. For $\bar{u}<r_{f}$, the left-hand side of (A33) is smaller than the right-hand side, so for $\bar{u}<r_{f}$ it solves only for $\bar{u}=0$, corresponding to $X_{0}^{*}=S_{0}$.

To compare $X_{0}^{*}$ for a negative surplus and $\nu \rightarrow \infty$ with the one in the mean-shortfall model for $\lambda \rightarrow \infty$, observe that in the mean-shortfall case, the limiting first-order condition is like Equation (A33), but with the right-hand side equal to zero. With $\bar{u}^{-}>r_{f}$, for a negative surplus the right-hand side of (A33) is negative, so the limiting $\bar{u}^{\infty}$ is larger than in the mean-shortfall model. From the definition of $\bar{u}$, it follows that the limiting $X_{0}^{*}$ is smaller than in the mean-shortfall model.

\section{Acknowledgments}

The authors thank Guus Boender, Cees Dert, and two anonymous referees for useful comments and discussions.

\section{References}

Basak, S., A. Shapiro. 2001. Value-at-risk based risk management: Optimal policies and asset prices. Rev. Financial Stud. 14(2) 371-405. 
Berkelaar, A. B., R. Kouwenberg. 2000a. Dynamic asset allocation and downside-risk aversion. EI-report 2000-12/a, Erasmus University, Rotterdam, The Netherlands.

Berkelaar, A. B., R. Kouwenberg. 2000b. Optimal portfolio choice under loss aversion. Econometric Institute Report EI 2000-08/A.

Berkelaar, A. B., R. Kouwenberg, S. Zhang. 2000. A primal-dual decomposition algorithm for multiple stage stochastic convex programming. Working paper, Erasmus University, Rotterdam, The Netherlands.

Berkelaar, A. B., C. Dert, B. Oldenkamp, S. Zhang. 2002. A primal dual decomposition-based interior point approach to two-stage stochastic linear programming. Oper. Res. 50(5) 904-915.

Bertsimas, D., G. J. Lauprete, A. Samarov. 2004. Shortfall as a risk measure: Properties, optimization and applications. J. Econom. Dynamics Control. 28 1353-1381.

Birge, J. R., F. Louveaux. 1997. Introduction to Stochastic Programming. Springer-Verlag, New York.

Boender, C. G. E. 1997. A hybrid simulation/optimisation scenario model for asset/liability management. Eur. J. Oper. Res. 99 126-135.

Cariño, D., T. Kent, D. Myers, C. Stacy, M. Sylvanus, A. Turner, K. Watanabe, W. Ziemba. 1994. The Russell-Yasuda Kasai model: An asset/liability model for a Japanese insurance company using multistage stochastic programming. Interfaces 24 29-49.

Cass, D., J. Stiglitz. 1970. The structure of investor preferences and asset returns, and separability in portfolio allocation: A contribution to the pure theory of mutual funds. J. Econom. Theory 2 122-160.

Consigli, G., M. A. Dempster. 1998. Dynamic stochastic programming for asset-liability management. Ann. Oper. Res. 81 131-162.

Dert, C. L. 1998. A dynamic model for asset liability management for defined benefit pension funds. W. T. Ziemba, J. M. Mulvey, eds. Worldwide Asset and Liability Modeling. Cambridge University Press, Cambridge, UK, 501-536.

Dert, C., B. Oldenkamp. 2000. Optimal guaranteed return portfolios and the casino effect. Oper. Res. 48(5) 768-775.

Harlow, W. 1991. Asset allocation in a downside-risk framework. Financial Analysts J. 47(5) 28-40.

Hiller, R. S., J. Eckstein. 1993. Stochastic dedication: Designing fixed income portfolios using massively parallel Benders decomposition. Management Sci. 39(11) 1422-1438.

Ingersoll, J. E. 1987. Theory of Financial Decision Making. Rowman and Littlefield Publishers, Inc., Lanham, MD.
Jorion, P. 2000. Value at Risk: The New Benchmark for Managing Financial Risk, 2nd ed. McGraw-Hill, New York.

Kahneman, D., A. Tversky. 1979. Prospect theory: An analysis of decision under risk. Econometrica 47(March) 263-291.

Kritzman, M., D. Rich. 1998. Beware of dogma: The truth about time diversification. J. Portfolio Management 24(4) 66-77.

Markowitz, H. M. 1959. Portfolio Selection. John Wiley and Sons, New York.

Merton, R. C. 1969. Lifetime portfolio selection under uncertainty: The continuous-time case. Rev. Econom. Statist. 51(August) 247-257.

Merton, R. C. 1990. Continuous-Time Finance. Basil Blackwell, Inc., Padstow, UK.

Merton, R. C., P. A. Samuelson. 1974. Fallacy of the log-normal approximation to optimal portfolio decision-making over many periods. J. Financial Econom. 1 67-94.

Mulvey, J. M., A. E. Thorlacius. 1998. The Towers Perrin global capital market scenario generation system. W. T. Ziemba, J. M. Mulvey, eds. Worldwide Asset and Liability Modeling. Cambridge University Press, Cambridge, UK, 286-312.

Rockafellar, R., S. Uryasev. 2000. Optimization of conditional value-atrisk. J. Risk 2(3) 21-41.

Samuelson, P. A. 1994. The long-term case for equities, and how it can be oversold. J. Portfolio Management 21(1) 15-24.

Sethi, S. P. 1998. Optimal consumption-investment decisions allowing for bankruptcy: A survey. W. T. Ziemba, J. M. Mulvey, eds. Worldwide Asset and Liability Modeling. Cambridge University Press, Cambridge, UK, 397-426.

Sharpe, W. F. 1998. Morningstar's risk-adjusted ratings. Financial Analysts J. 54(4) 21-33.

Siegmann, A. H. 2003. Optimal financial decision making under loss averse preferences. Ph.D. thesis, Vrije Universiteit Amsterdam, Amsterdam, The Netherlands.

Sortino, F., R. Van der Meer. 1991. Downside risk: Capturing what's at stake in investment situations. J. Portfolio Management 17(4) 27-31.

Tversky, A., D. Kahneman. 1992. Advances in prospect theory: Cumulative representation of uncertainty. J. Risk Uncertainty 5 297-323.

Ziemba, W., J. M. Mulvey, eds. 1998. Worldwide Asset and Liability Modeling. Cambridge University Press, Cambridge, UK. 